Published in final edited form as:

Behav Processes. 2013 February ; 93: 111-115. doi:10.1016/j.beproc.2012.11.009.

\title{
Chasing Sounds
}

Julie J Neiworth, Ph.D.

Dept of Psychology, Carleton College

\begin{abstract}
Prior work with Wright and others demonstrated that rhesus monkeys recognized the relative relationships of notes in common melodies. As an extension of tests of pattern similarities, tamarins were habituated to 3-sound unit patterns in an $\mathrm{AAB}$ or $\mathrm{ABB}$ form that were human phonemes, piano notes, or monkey calls. The subjects were tested with novel sounds in each category constructed either to match the prior pattern or to violate the prior habituated pattern. The monkeys attended significantly more to a violation of their habituated pattern to a new pattern when human phonemes were used, and there was a trend difference in attention toward pattern violations with melodies. Monkey call patterns generated a variety of behavioral responses, were less likely to show habituation, and did not generate a strong attention reaction to changes in the patterns. Monkeys can extract abstract rules and patterns from auditory stimuli but the stimuli, by their nature, may generate competing responses which block processing of abstract regularities.
\end{abstract}

Recent research on auditory perception in monkeys has uncovered interesting and sophisticated cognitive processing to naturalistic stimuli and more complex melodies. In earlier work, several monkey species including capuchin (Cebus apella) and rhesus (Macaca mulatta) representing New World and Old World groupings struggled to discriminate simple auditory dimensions like absolute pitch in discrimination tasks. This outcome suggested a strong modality asymmetry between visual and auditory simple stimuli in monkeys (Cowey, 1968; D'Amato \& Salmon, 1982; Wegener, 1964; Poremba, Saunders, Crane, Cook,

Sokoloff, \& Mishkin, 2003). More recently, researchers have found pitch-selective neurons in the auditory cortex in a New World primate, the common marmoset (Callithrix jacchus). Using single-unit extracellular recordings, Bendor and Wang (2005) found a cortical region near the anterolateral border of primary auditory cortex in the marmoset containing neurons that respond significantly to pure tones. Recent human imaging studies have revealed a cortical pitch processing region anterolateral to primary auditory cortex (Penagos, Melcher, \& Oxenham, 2004). These findings suggest similar brain processing of pitch in humans and at least some monkey species. They also suggest that monkeys may not readily demonstrate a grasp behaviorally to learn differences between very simple pure tones although their brains code for the differences. Alternatively, more complex tonal sequences which match better monkeys' natural calls and sounds may solicit more cognitive processing and a demonstration of their cognitive prowess with auditory stimuli.

Constructing an auditory sequence with absolute changes among the tones defines a melody. The more abstract structure of a melody is the relative relationship, the rise and fall of

(C) 2012 Elsevier B.V. All rights reserved.

Correspondence concerning this article should be sent to Julie J Neiworth, Dept of Psychology, Carleton College, Northfield, MN 55057.

Publisher's Disclaimer: This is a PDF file of an unedited manuscript that has been accepted for publication. As a service to our customers we are providing this early version of the manuscript. The manuscript will undergo copyediting, typesetting, and review of the resulting proof before it is published in its final citable form. Please note that during the production process errors may be discovered which could affect the content, and all legal disclaimers that apply to the journal pertain. 
individual tones with respect to each other. When a melody is transposed or when its absolute frequencies are changed but its frequency relations are preserved, humans perceive the transposed melody as similar to the original one because the melody contour is identical. Perception of such relationships between frequencies, or relative pitch perception, is prominent for humans from early stages of development ( Chang \& Trehub, 1977; Demany \& Armand, 1984; Trehub, Bull, \& Thorpe, 1984; Trehub, Thorpe \& Morrongiello 1987). But is this a primate-general ability?

Early studies of melody contours by D'Amato (1988) and D'Amato and Salmon (1982 and D'Amato and Salmon (1984) in cebus monkeys suggested the use of absolute cues to discriminate melodies and thus a restricted use of relative relationships because the monkeys seemed to use local cues for discrimination. Others found similar use of absolute cue strategies in rhesus monkeys (Moody, May, Cole and Stebbins, 1986). However, Izumi (2001) trained 3 Japanese monkeys (Macaca fuscata) to detect changes from rising to falling contours of 3-tone sequences and the monkeys were able to transfer relative pitch perception to novel octave-shifted sequences, if only within the absolute frequency range of the training phase.

Before the Izumi study, Wright and I and several colleagues explored rhesus monkeys' understanding of relative relationships among tones (Wright, Rivera, Hulse, Shyan, \& Neiworth, 2000) by constructing melodies that were recognized by humans to have strong gestalt or grouping properties (i.e., childhood songs like "Happy Birthday"), and by testing monkeys who had been trained to judge whether a sound was the same or different from previously heard sounds. Same-different judgments are considered more abstract in that the idea of sameness is typically based on category membership in which many items may be slightly different but they carry a common structure or organization. Same/different discriminations preclude the use of absolute unique singular features, unless one focuses on absolute differences in the decision, which is antithetical to "sameness". We found that the two rhesus monkeys in the study could readily generalize, or identify as "same" strong melodies that were transposed 1 or 2 octaves from the original presentation. This generalization failed when the melodies were constructed more randomly, and thus a strong internal structure was needed in order for the monkeys to "hear" transposed melodies by whole octaves as the same as the original melodies. This study demonstrated that monkeys can treat melodies as whole units and can generalize particular shifts in the same way that humans do. It also showed several limitations by monkeys in doing so; both based on the internal structure of the melody, and based on the type of shift that was imposed.

Since my contribution to some of this work with Wright, I have often considered how monkeys process auditory stimuli we present to them. The fact that the structure of auditory stimuli influences strongly how monkeys perceive and categorize sounds suggests that there are potentially resources used by monkeys for listening to monkey calls (their own communication system), for naturalistic sounds (like other species' communication sounds), and for music that have gone on untapped and not measured in our experiments thus far. Matthews and Snowdon (2011) found that cotton top tamarins (Saguinus oedipus), a New World monkey species that I also study, recognize the "long" calls of tamarin relatives for approximately 4 years, as evidenced by lower arousal levels to their calls than to other tamarins they may have heard during the same time period. The long call, constructed as a rising series of continuous sound, must be processed very particularly for relatives of monkeys for the habituated reaction to be maintained to individuals' calls. Snowdon also examined tamarins' reactions to cello music composed to match agitated monkey calls or relaxed monkey calls, and found the similar emotional reactions of agitation and relaxation when the two types were played (as described in Dingfelder, 2009). This finding suggests that music constructed with a structure similar to conspecific calls may be processed 
similarly, showing an emotional link connected to particular structures of sounds which may have evolved early in primates' history.

Another scientific event forced my hand to return to testing auditory stimuli when there was a retraction of a study originally published in 2002 by Hauser, Weiss, and Marcus in which tamarins showed an attentional reaction similar to very young infants to changes in phoneme (i.e., consonant-vowel sounds) sequences. In particular, in both infants and tamarins, when they were habituated to a particular sequence like AAB (or wi wi di), and that sequence was represented by very different local phoneme events (like ga ga ti, li li la, etc), they noticed when the abstract pattern changed to $\mathrm{ABB}$ more so than when novel phonemes were presented in the same AAB pattern. This finding suggests that tamarins and humans share an ability to extract abstract patterns from human language sounds, and thus the perception and judgment of the regularity of human phoneme patterns is not something that is humanspecific. Two issues require a response to this study: 1) are the findings replicable, given there is a question about missing data that led to the retraction in 2010, and 2) exactly how are the tamarins processing the phoneme sequences? Taking into consideration the past work on melodies and relative relationships, one would expect tamarins to be able to form an abstract category of an $\mathrm{AAB}$ or $\mathrm{ABB}$ pattern, but the ability to form this may be related to the significance, the salience, or the psychological "strength" of the structure of the relative pieces. Recall that randomly constructed melodies using the same notes as melodies that humans readily remember were not generalized by rhesus monkeys in the former Wright et al (2000) study. Music structured like monkey calls did provoke similar arousal reactions to the calls themselves. Thus one would expect differences in success and failure to generalize similar sequences and to note different sequences in melodies, monkey calls, and human language by tamarins.

I have spent a year testing habituation and dishabituation by tamarins to human phoneme sequences, melodies, and edited monkey calls. In every auditory case, structures resembling $\mathrm{AAB}$ and $\mathrm{ABB}$ were made. In every case, half of the subjects were exposed to an $\mathrm{AAB}$ sequence repeatedly, which changed locally within each session and across sessions in terms of cues used to make the sequence. The other half of the subject pool was exposed to ABB sequences repeatedly. Then a test of a novel sequence matching their habituated structure (another AAB when they had heard AAB's in training, for example) and a test of a novel sequence inconsistent with their habituated structure (an ABB sequence when they had heard AAB's in training, for example) were presented. Arousal and attention to the novel sequences were measured by eating behavior. Past research across a variety of mammalian species has indicated that food eating behavior can be altered by novel or stressful stimulus presentations (for a review, see McSweeney and Swindell, 1999), and human infants will decrease high amplitude sucking behavior to novel languages but increase it to something more familiar (Moon, Cooper, and Fifer, 1993, for example). The analyses presented here are based on eating behavior with short-term limited exposure to a preferred food, which increases when subjects experience lowered arousal due to habituation and decreases substantially when subjects are attending to novel items ${ }^{1}$. Look rate behavior was videotaped for every training and test sequence and is being coded and scrutinized for interrater reliability, and will be published in another article, once the coding is complete.

\footnotetext{
${ }^{1}$ Look rate behavior was videotaped for every training and test sequence and is being coded and scrutinized for inter-rater reliability, and will be published in another article once the coding is complete.
} 


\section{Methods}

\section{Subjects}

The subjects were 16 adult-aged cotton top tamarins, (Saguinus oedipus) housed in pairs or triplet groups in 3 different monkey colony rooms in the animal facility at Carleton College. There were 10 females and 6 males in the original phoneme condition, but in one case (a female), the test was not recorded properly, so only 15 subjects' data were analyzed. Three females died over the course of the study, leaving 13 monkeys in the music condition, and in the final monkey call condition. The ratio of females to males in the final two conditions was 7: 6. None of the subjects had participated in an auditory discrimination experiment before this study, although they had heard humans talking and monkey calls of relatives and unfamiliar tamarins in the colony during their lifetimes. The age range of the monkeys was 5 (Egret) to 19 (Quince), with adult onset occurring around 21 months. All subjects had been adults for at least 3 years before the study began, so the age range only captures a difference in adult ages, not a developmental difference.

The monkeys were monkey-family reared in laboratory settings and had been socially housed in pairs in seven different $0.85 \times 1.50 \times 2.30 \mathrm{~m}$ cages, with the cages visually separated by opaque sheets. The subjects were on a 12-hr light-dark cycle and had free access to water. All animals were maintained on a complete diet consisting of a yogurt and applesauce breakfast, a lunch of Zupreem Marmoset chow, fruits and vegetables, and a protein snack (e.g., eggs, hamburger, mealworms) daily. The protocol and care of the monkeys were approved by the Institutional Animal Care and Use Committee, and the monkeys were inspected regularly by USDA and their care met consistently animal welfare assurances.

\section{Materials}

There were 3 sets of sounds used: phoneme sequences, musical note sequences, and monkey call sequences. Some properties were the same for each sequence. Those common properties are described first, with the particular characteristic of each sound type described separately.

For each type of sequence, there were 3 sound units, separated by empty pauses. Each sound unit was edited in Audacity to last $0.5-0.7$ seconds in duration, as was typical for spoken phonemes in the English language. Each sound unit started on a whole second, so for example, the first sound started at the 0 -second mark, followed by the second sound at the 1.0 -second mark, followed by the $3^{\text {rd }}$ sound at the 2.0 -second mark. Thus there was a $0.3-$ 0.5 second pause between sound units within a sequence due to the length of the fully pronounced phoneme, and consequent matching musical note or monkey call. Each 3-sound sequence was played 4 times to form a trial. Within a trial each sequence presentation was started on a 5.0-second mark, so the first sequence was started at 0 seconds, the second sequence, at 5.0 seconds, and the third sequence at 10.0 seconds, the $4^{\text {th }}$ at 15 seconds. Thus there was a delay of approximately 2.5 seconds which separated each 3 -unit sequence presentation. Each trial lasted about 17-20 seconds in duration. All timing variations were matched exactly across the 3 sound stimuli types (phonemes, melodies, and monkey calls) such that timing itself within a sequence or across sequences was not a discriminable factor across sets. Half of the subjects heard an AAB sequence, played 4 times per trial, and half of the subjects heard an $\mathrm{ABB}$ sequence, played 4 times per trial. The $\mathrm{ABB}$ sequence used the same sound units as the $\mathrm{AAB}$ sequence; only the pattern was altered.

For phoneme sequences, each phoneme was constructed of a consonant-vowel combination. The phonemes used in the training set included plosive consonants ( $, \mathrm{b}, \mathrm{d}, \mathrm{k}, \mathrm{t}$ ) and nasal consonants (m, n), as well as closed front (i) and closed back (u) vowels. So for example, the AAB patterns used in training included di di bu, pu pu ki, mu mu ni, pi pi gu, bi bi du, nu nu 
mi, du du ki, pi pi tu, ti ti ku, and ku ku di. The ABB sequences used the same phonemes except in the ABB pattern, for example, di bu bu for the first sequence. The test phonemes were constructed with novel glide consonants ( $w, r, j$ ) or liquid consonants (l), and a central open vowel (a) or a closed front vowel (i). The test phoneme sequences were la la ri and wa ji ji. All phoneme sounds were obtained from Hauser and were the female "Judy" voice used in the original 2002 study. The sequences were built using Audacity at Carleton College. They were presented using Audacity through an Apple MacBook Pro with external speakers.

The melody sequences were composed of 3 notes formed by the software piano included in Garage Band software. Notes were selected from frequencies from C ( $5^{\text {th }}$ octave) (523.251HZ) to C ( $7^{\text {th }}$ octave) $(2093 \mathrm{HZ})$ to match to some degree the normal pitch of monkey calls of this species, which is rather high-pitched. Within a melody sequence, each note was selected to be within an interval ranging from three to five white notes from the former note. The AAB patterns used in training included CCE, AAD, EEB, FFA, DDG, AAE, CCF, AAC, GGB, and EEB. Some of these sequences were in the $5^{\text {th }}, 6^{\text {th }}$ or $7^{\text {th }}$ octave, but within that octave, the notes were within 5 white notes of each other on a piano. The ABB patterns used the same notes only matched the new pattern, so for example, the first sequence was CEE. The test sequences included GDD and FFC, both in octaves not used for those notes in previous sequences, and both descending in pattern while all training patterns were rising or ascending pitches. All sequences were formed using Audacity software, and played through Audacity on the same Apple MacBook Pro with the same external speakers.

The monkey calls were recorded in $10-15$ minute sessions by two undergraduate researchers during feeding times and also during a time of stress, when a confederate student donned a full face mask and marched around the colony rooms to solicit barks and warning calls. The calls were then downloaded to Audacity to be edited into 0.5-0.7 second sound units for use in sequences to match the length of the phonemes and musical notes. Attempts were made to parse at natural communicative sounds. The researchers could readily identify different "long" calls, barks, chirps, and screeches used often to indicate searches, warning calls, and food-related calls. The monkey calls that were edited included 4 different "long" calls that consisted of ascending continuous calls, 4 different monkey barks which are shorter bursts, 2 screeches evoked just before scattering following an intruder, and several chirping calls solicited by feeding times, each with multiple chirping sounds at different pitches. These were coded and mixed such that AAB training sequences included Bark1Bark1-Chirp1, or Bark2-Bark2-Screech1, for example. ABB sequences used the same sound units but matched the appropriate sequencing, for example, the first sequence was Bark1Chirp1-Chirp1. The test call sequences were composed of a High-Low-High (HLH) call, a double chirp (DC1), a long call (LC1) and a double-bark (DB1) which had not been used during training. They were HLH-DB1-DB1 and LC1-LC1-DC1.

\section{Procedure}

There were 3 sound conditions consisting of habituation ( 5 sessions) and a test ( 2 trials) conducted in the same series for all subjects: Phoneme habituation and testing, Melody habituation and testing, Monkey calls habituation and testing. For each condition, subjects were presented 2 trials of auditory sequences in each session for 5 consecutive sessions. Each trial consisted of 4 presentations of the same auditory sequence. Before each trial began, undergraduate researchers would position a single digital videocamera on a tripod in front of each cage in which a pair or triplet of monkeys was housed. The primary investigator (PI) moved the laptop computer and speakers on a small cart behind the cages in the room to a corner of the room. On her cue, the undergraduate researchers would simultaneously enter the cages and deposit 10 Frosted Cheerios in each food bowl and exit the cage. They would immediately stand behind their cameras and begin recording. The PI 
would then play the first trial, which consisted of 4 presentations of the same ABB or AAB sequence, depending upon the assignment for habituation for the monkeys in the room. Each trial lasted about 17-20 seconds, at which point the undergraduate researchers would immediately re-enter the cages and report how many Frosted Cheerios remained. Monkeys could eat Frosted Cheerios while listening to the stimuli, but if they were surprised or were attending closely to the stimuli, their orienting behavior typically prevented them from engaging in eating. Thus if a monkey was really interested in attending to the stimulus, he may have 10 Cheerios remaining and may not have eaten any during the trial. The researchers would then repeat this process for a second trial to complete the session. When recording, the undergraduate researchers would focus on the body and head posture. Because rewards were being offered, the monkeys usually traveled to the food shelf and so were together during the recording period. The data reported here are the rates of eating Cheerios during the sound sequence presentations, recorded as a percentage of the total eaten. If no Cheerios were eaten of the 10 offered, a $0 \%$ eaten score would be entered. If 3 Cheerios of the 10 were eaten, a $30 \%$ eaten score would be entered.

Each pair of subjects was exposed to the training pattern (either ABB or AAB) for 5 sessions, with 2 trials presented per session. In the $5^{\text {th }}$ session, 2 training trials were presented, followed by 2 test trials. The test trials were composed of one trial in which the sounds were novel and the pattern was novel (i.e., ABB if the subjects had habituated to $A A B)$, and one trial in which the sounds were novel but the pattern was the same one from habituation (AAB if the subjects had habituated to $\mathrm{AAB}$ ). The same data (look rates as indicated from video of body and head posture, and amount of Cheerios eaten) were recorded in the test trials.

For each condition, half of the subjects were habituated to $\mathrm{AAB}$ and the other half, to ABB. In each room, all subjects housed in that room were habituated to one type of sequence. Across rooms the sequence used for habituation varied. The phoneme habituation and test occurred in December, 2011. The melody habituation and test occurred in March of 2012. The monkey call habituation and test occurred in August, 2012. At least 3 months of time separated each habituation and test.

\section{Results}

For each condition (phoneme, melody, monkey call) habituation was graphed as a function of average percentage of food eaten across the 5 habituation sessions. Figure 1 shows the rate of habituation to phoneme sequences, to melody sequences and monkey calls, respectively.

In each condition, there was a change to more eating in the last session as compared to the first. For phoneme habituation, a best-fitting linear trend indicates a $1 \%$ increase per session, with the eating rate starting at $32.4 \%\left(\mathrm{R}^{\wedge} 2=0.77\right)$. In contrast, for melody habituation, the eating rate was suppressed in Session $1(10.7 \%)$. Anecdotally, we observed that the monkeys seemed more agitated and attentive initially to the melody sequences. A bestfitting linear trend indicates a 5\% increase in eating per session $\left(\mathrm{R}^{\wedge} 2=0.72\right)$. The monkey call sequence was the only auditory set that generated a sensitization effect because Session 2 generated a much lower rate of eating (12.86\%) than Session 1 (22.86\%). Overall, the best-fitting linear trend for data from Figure 3 shows a $3 \%$ increase in eating across sessions, but with only $38 \%$ of the variance accounted for by the line $\left(\mathrm{R}^{\wedge} 2=0.38\right)$. The rates of habituation to the same number of exposures of the same duration sequences varied depending upon the nature of the sound. Phoneme sequences and melody sequences produced more consistent slow habituation for which increasing functions were noted, and 
most of the variance was accounted for by best-fitting lines. The monkey call sequences produced the most varied arousal, with a fairly poor fit from a linear trend analysis.

A separate repeated measures analysis of variance (ANOVA) was conducted using average percent food eaten per subject as the dependent variable to 3 trial types (trained set, test with same pattern, test with different pattern) for each condition (Phoneme, Melody, Monkey calls). Figure 2 shows the mean and variance, represented as standard deviations, to the last 2 training trials, the test with the same pattern, and the test with the different pattern, across these 3 conditions.

The ANOVA comparing the amount eaten across the last 2 training trials and the two test trials in the Phoneme condition was significant, $\mathrm{F}(2,14)=4.04, \mathrm{p}=0.04$. By pairwise comparisons between the trained set and the two tests, there was significantly less eaten when the test violated the prior habituated pattern $($ mean $=24.58 \%)$ as compared to the amount eaten to the trained set $($ mean $=36.78 \%), p=0.02$. The difference in amount eaten between the trained set and the test which maintained the same pattern was not significant, $p$ $=0.12$. Thus the monkeys hesitated more and subsequently ate less when presented a novel sequence that also violated the pattern to which they had habituated when the stimuli were human phonemes.

The ANOVA comparing the amount eaten across training and the two tests in the melody condition was not significant $(\mathrm{p}=0.28)$. By pairwise comparisons between the trained set and the two tests, there was a trend difference between eating rates to the novel test which violated the prior pattern $($ mean $=24.29 \%$ eaten, $\mathrm{p}=0.077$ ) and the trained set $($ mean $=$ $32.5 \%$ ). All other comparisons were not significant. The ANOVA testing the amount eaten against the trained and test conditions in the monkey call condition was also not significant $(\mathrm{p}=0.41)$ and none of the pairwise comparisons between the trained set and the two tests was significant nor a trend.

\section{Discussion}

This experiment tested whether tamarins habituate to regular repeated patterns of auditory stimuli such that a violation of the former pattern produces dishabituation. It is clear from these results that tamarins can extract an abstract regular pattern, but the extent to which they can do so depends upon the nature of the auditory stimuli. With human phonemes spoken by a female voice, the tamarins showed a relaxed response from the first presentation, and only marginally habituated more to the sounds over a 5-session training period, as evidenced by a $1 \%$ average increase in eating treats per session, and a rise from $30-36 \%$ eating time to presentations. More importantly though, when the pattern of consonant-vowel units changed (either from $\mathrm{AAB}$ to $\mathrm{ABB}$ or from $\mathrm{ABB}$ to $\mathrm{AAB}$ ), the tamarins showed a significant drop in eating during stimulus presentation. Their need to orient to the new pattern and process it as distinctly different consequently yielded less time to eat the treats.

But how should we interpret this finding? Does it suggest that tamarins show a sensitivity to human phoneme patterns shared with very young human infants? Does it suggest a more general sensitivity to regularized patterns of auditory stimuli that may be applied to any sounds and also happens to work with human phonemes? By comparing dishabituation outcomes across several auditory categories and by considering the relevance of the auditory source to the subject and the rate of habituation by the source, it should be possible to narrow the possible explanations for this phoneme pattern sensitivity effect.

First, the same level of dishabituation, as evidenced by decreased eating rates, was not found for melodies and monkey calls with the same strength as it was found for human phonemes. 
Thus, the phoneme pattern sensitivity that the monkeys showed is not based on a general statistical pattern recognition strategy applied to any pattern of auditory stimuli equivalently. Rather, a violation of a habituated auditory pattern generates more attention by tamarins to human phonemes than to melodies, and less to novel changes in monkey call sequences.

Why can tamarins sometimes note pattern changes in complex auditory stimuli and sometimes not? One clue may reside in the habituation rates to each class of stimuli. The phoneme sequences did not generate a strong initial attention reaction. In contrast the melody sequences suppressed eating rates to $10 \%$, a full $20 \%$ lower than the reaction tamarins had to phonemes. It's possible that anxious and agitated responses block cognitive processing of the pattern of events more than the events themselves. This would explain more arousal to new phoneme patterns following phoneme habituation, and a more ambiguous arousal reaction to melody pattern changes because melodies, and possibly the notes themselves, originally produced more arousal.

The most variability in response happened during monkey call habituation, in which monkeys reacted with suppressed eating and increased eating in a fairly random pattern, with only slight habituation noted (3\% increase per session of eating, but only $38 \%$ of the data accounted for by this linear analysis). I can report that the monkey calls had to be edited to fit the time window to match the other stimuli but strong effort was made to select full complete monkey call sound bytes to the extent that we recognize full complete calls and can code specific calls by our own hearing of them and their elicited reactions. Still, the conspecific calls we selected were mixed in ways which produced triplets of calls not normally uttered so close temporally, i.e., a food-related call coupled with territorial barks or screeches. Anecdotally, the monkeys behaved stereotypically to the calls by running in circles and scattering to screeches, and to replying with long calls to partial long calls played to them. In this case, the monkeys' cognitive processing was divided between processing what they heard and reacting to it. In a limited capacity model for attention, this should produce poor quality cognitive processing of more abstract elements, such as how the individual sounds produce a pattern.

The very different attention demands placed on the tamarins by the different categories of auditory stimuli produced the successes and failures by monkeys to notice pattern violations in this study. Human language is heard all the time and does not generate a strong alerting response, thus more subtle qualities like the patterns of sounds being perceived may be more easy to note and remember. Melodies made from piano notes are more foreign to the tamarins and, in this case, were not necessarily constructed to make a strong gestalt unit. The tamarins noted when the pattern changed, but less well than with human phonemes. The monkey calls cobbled together as $\mathrm{ABB}$ or $\mathrm{AAB}$ patterns used communicative signals in novel presentations to the monkeys, and rather than note the pattern, they reacted to the information contained in the discrete units (long call response, or territorial or hiding response). The lesson from this study, as was true of many of Wright's visual concept learning experiments, is that testing a variety of stimuli is very important, as is considering the meaningfulness or relevance of different types of stimuli to the monkey subject. A natural monkey call, if presented clearly, may induce single unit processing due to the relevance to the subject and this processing may block subtle or abstract cognitive processing, especially if the sound invokes a plan to action that divides the monkey's attention. In contrast, a limited simple auditory stimulus may not gain enough attention by the monkey to show learning or discrimination. To determine whether monkeys can notice pattern regularities or gestalt wholeness in melodies, calls, music, or language requires a consideration of the context generated by the sounds and the meaning the sounds provoke. 


\section{Acknowledgments}

This research was funded in part by an NIH AREA grant \# R15DH072751 to the author. The author would like to thank Anthony A Wright for his collaborations and creativity. She would also like to recognize the following undergraduate researchers who helped with this project: Dee Dee Rupert, Rae Wood, Owen Alldritt, Deborah Tan, Jordan Palacios, Caleb Hyde, Sofia Levinson, and Evia Zack, Carleton College.

\section{References}

Bendor D, Wang X. The neuronal representation of pitch in primate auditory cortex. Nature. 2005; 436:1161-1165. [PubMed: 16121182]

Chang HW, Trehub SE. Auditory processing of relational information by young infants. Journal of Experimental Child Psychology. 1977; 24:324- 331.

Cowey, A. Discrimination. In: Weiskrantz, L., editor. The analysis of behavior change. New York: Harper \& Row; 1968.

D'Amato MR, Salmon DP. Tune discrimination in monkeys (Cebus paella) and in rats. Animal Learning \& Behavior. 1982; 2:126-134.

Demany L, Armand F. The perceptual reality of tone chroma in early infancy. Journal of the Acoustical Society of America. 1984; 76:57- 66. [PubMed: 6747112]

Dingfelder S. Monkeys mostly like monkey music. APA Monitor. 2009 Nov 10.2009:10.

Hauser MD, Weiss D, Marcus G. Rule learning by cotton-top tamarins. Cognition. 2002; 86:B15-B22. [PubMed: 12208654]

Izumi A. Relative pitch perception in Japanese monkeys (Macaca fuscata). Journal of Comparative Psychology. 2001; 115:127-131. [PubMed: 11459159]

Matthews S, Snowdon CT. Long term memory for calls of relatives in cotton top tamarins. Journal of Comparative Psychology. 2011; 125:366-369. [PubMed: 21574684]

McSweeney FK, Swindell S. General-process theories of motivation revisited: The role of habituation. Psychological Bulletin. 1999; 125:437-457.

Moody DB, May B, Cole DM, Stebbins WC. The role of frequency modulation in the perception of complex stimuli by primates. Experimental Biology. 1986; 45:219-232. [PubMed: 3732498]

Moon C, Cooper RP, Fifer WP. Two-day olds prefer their native language. Infant Behavior and Development. 1993; 16:495-500.

Penagos H, Melcher JR, Oxenham AJ. A neural representation of pitch salience in nonprimary human auditory cortex revealed with functional magnetic resonance imaging. Journal of Neuroscience. 2004; 24:6810-6815. [PubMed: 15282286]

Poremba A, Saunders RC, Crane AM, Cook M, Sokoloff L, Mishkin M. Functional mapping of the primate auditory system. Science. 2003; 299:568-572. [PubMed: 12543977]

Trehub SE, Bull D, Thorpe LA. Infants' perception of melodies: The role of melodic contour. Child Development. 1984; 55:821-830. [PubMed: 6734320]

Trehub SE, Thorpe LA, Morrongiello BA. Organization processes in infants' perception of auditory patterns. Child Development. 1987; 58:741- 749. [PubMed: 3608646]

Wegener JG. Auditory discrimination behavior of normal monkeys. Journal of Auditory Research. 1964; 2:81-106.

Wright AA, Rivera JJ, Hulse SH, Shyan M, Neiworth JJ. Music perception and octave generalization in rhesus monkeys. Journal of Experimental Psychology: General. 2000; 129:291-307. [PubMed: 11006902] 


\section{Highlights}

- Tamarins were presented regular patterns of 3-sound sequences.

- When the pattern made of phonemes was violated, tamarins attended more.

- There was not a significant effect to changes in the patterns of melodies or monkey calls.

- Tamarins reacted overtly to individual monkey calls by barking or hooting back.

- Monkeys can extract rule-based patterns from auditory sequences of certain types. 


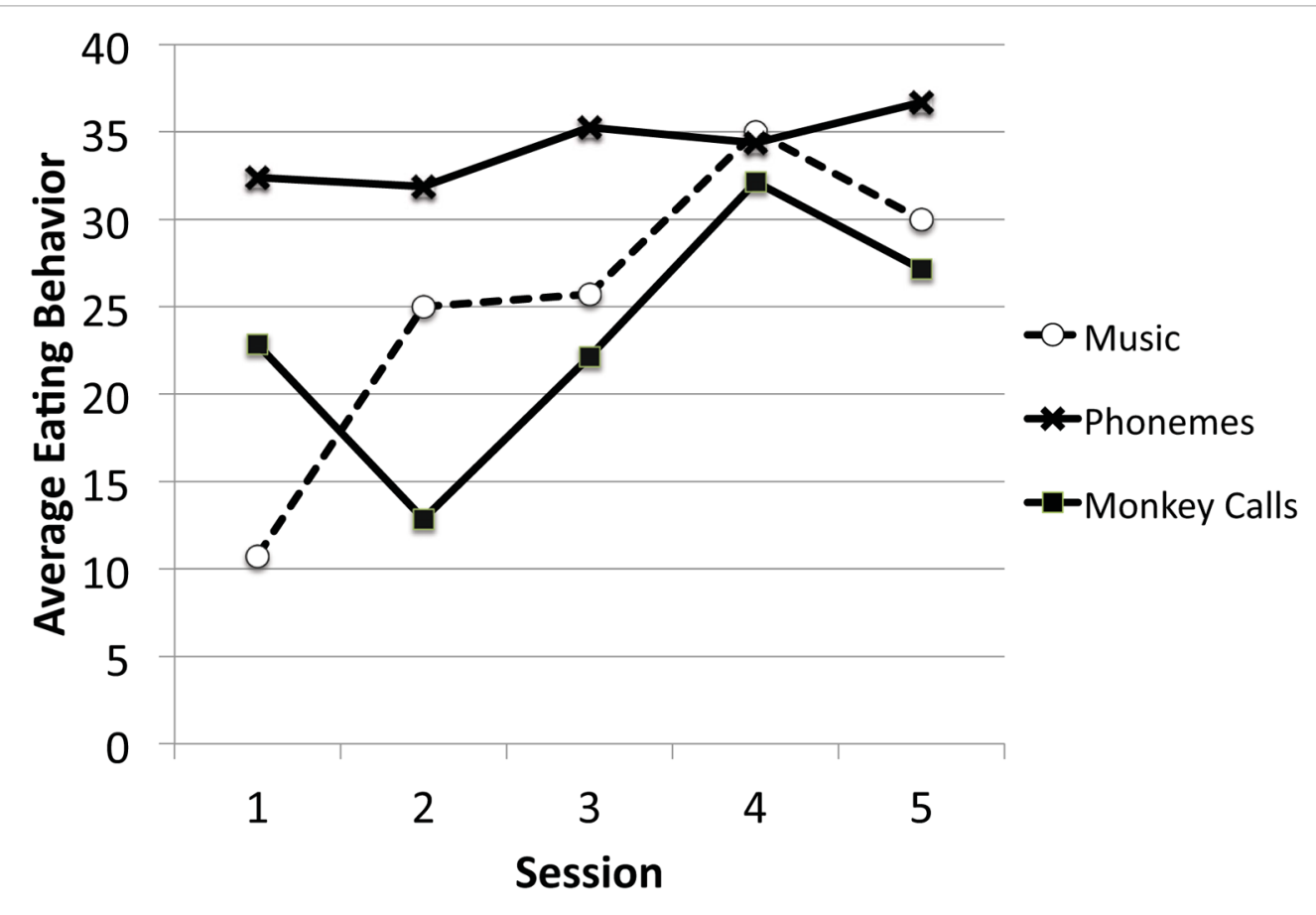

Figure 1.

Average percent Cheerios eaten during the phoneme sequence, the melody sequence, and the monkey calls sessions. 


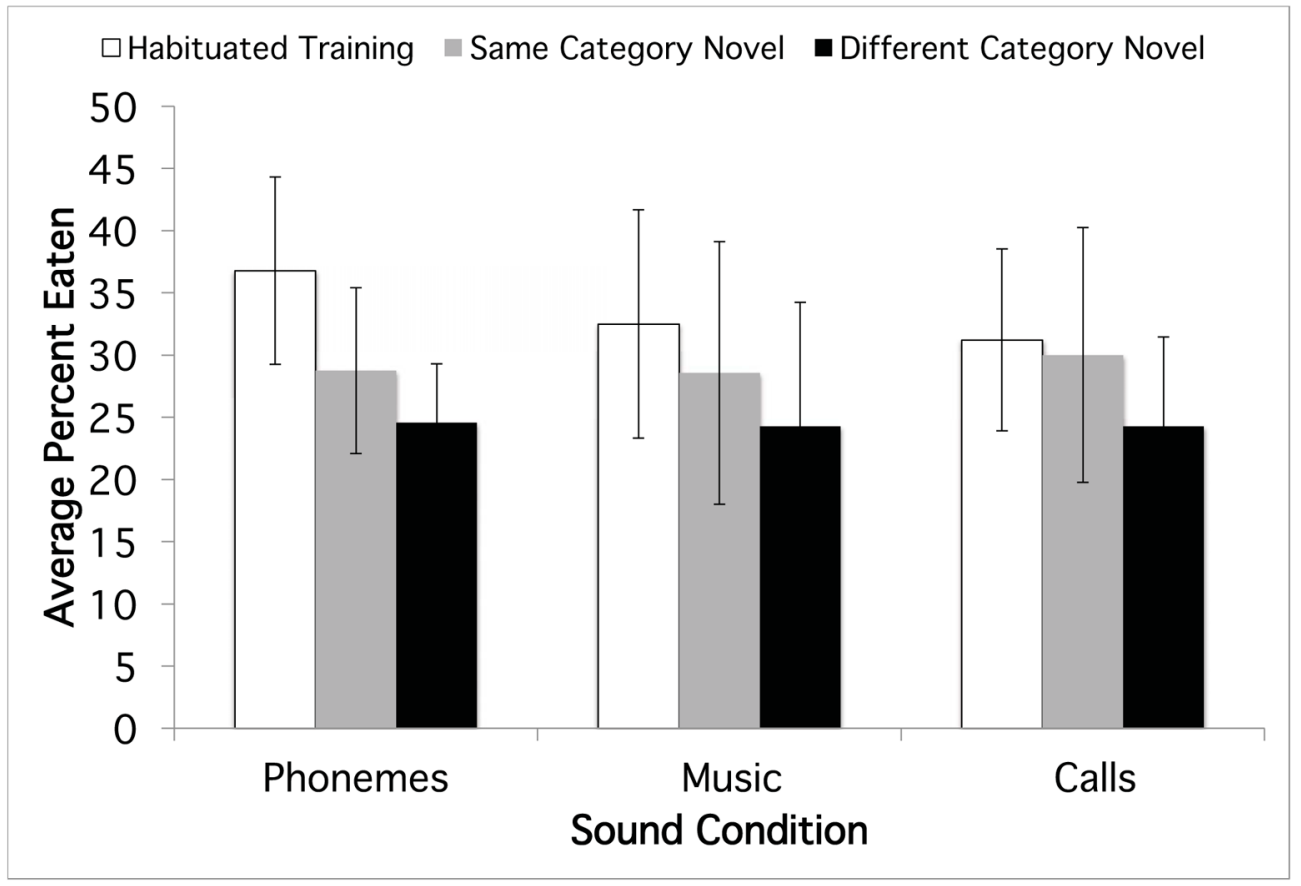

Figure 2.

Average amount eaten to the last 2 training trials in habituation compared to the two novel test trials, one which also violated the pattern (different category) and one which matched the habituated pattern (same category). Error bars show standard deviations. 